\title{
Resistor-Network Modeling of Ionic Conduction in Polymer Electrolytes
}

\author{
Jeffrey T Gostick ${ }^{1}$ and Adam Z Weber ${ }^{2, *}$ \\ ${ }^{1}$ McGill University, 3610 University St, H3A2B2, Montreal, QC, Canada \\ ${ }^{2}$ Lawrence Berkeley National Laboratory, 1 Cyclotron Road, MS 70-108B, Berkeley, CA 94720, \\ USA
}

\begin{abstract}
A resistor- and pore-network methodology is used to examine transport of ions in various ion-conducting polymers. The model is used to examine ion conduction in random and correlated (at the mesoscale) distributions of high and low conductive domains showing the impact that defects or different conduction modes have on overall effective conductivity and percolation. The specific case of Nafion is modeled where swelling is accounted for as well as a spatially varying conductivity within the nanodomains. The model is also used to investigate conduction in thin-films, where a substantial drop in conductivity is witnessed for films less than $50 \mathrm{~nm}$ thick. The model shows good agreement with experimental data and provides a methodology for efficient multiscale modeling of transport in ion-conducting polymers from the nanoscale through the mesoscale transport pathways to the observable macroscale properties.
\end{abstract}

Keywords: Proton-exchange membrane; pore-network modeling; polymer-electrolyte fuel cell; multiscale mathematical modeling; conductivity

*Corresponding Author: azweber@lbl.gov, 510-486-6308 


\section{Introduction}

Transport of ionic species through ion-conducting polymers, or ionomers, is a major consideration in the design of polymer-electrolyte fuel cells (PEFCs), redox flow cells, solar-fuel generators, and batteries. Ionic conduction, or lack thereof, is usually a significant source of efficiency loss in these devices, especially as one endeavors to simplify or scale systems [1]. For example, it is desired to operate PEFCs at low humidities and temperatures above $100^{\circ} \mathrm{C}$ to decrease system complexity and water management; however, an ionomer has yet to be developed that can conduct efficiently under such conditions. To minimize the overall cell ohmic resistance, the ionomer is made as thin as possible so that the effective conductance, a function of intrinsic conductivity and geometry, is sufficiently high. Such a strategy has a minimum threshold due to the need for mechanical stability and chemical durability of the membranes. The search for materials with high intrinsic conductivity and/or better mechanical properties is an ongoing task, including the development of composite structures composed of conductive and nonconductive elements [2-7].

Transport of ions within ionomers is an inherently multiscale phenomena, where ions are traversing the length of the membranes and interacting with dynamic nanophase separated domains that coalescence into transport pathways at the mesoscale [8]. Mathematical modeling is ideally suited for exploring ionomers due to their complexity and nonlinearity in their transport phenomena and even structural data. For PEFCs specifically, the proton-exchange membrane (PEM) plays an integral role in controlling the cell's ohmic losses as protons are subject to Ohm's Law. In addition, the PEM is a critical component in PEFC water management as it transports water and normally requires hydration for efficient proton conduction, thereby setting minimal cell humidity requirements. While there are efforts to develop PEMs that conduct efficiently at higher temperatures $\left(>100^{\circ} \mathrm{C}\right)$ and/or lower humidities, these are still in the laboratory setting [9].

The polymer of choice, Nafion ${ }^{\circledR}$, a random copolymer of polytetrafluoroethylene and 
polysulfonyl fluoride vinyl ether, serves as a benchmark material due to its remarkable proton conductivity and thermo-mechanical stability [10]. Absorption of water by the ionic groups creates nanodomains in the semi-crystalline polymer matrix through which ion and water transport occurs. Nanophase separation and water-uptake capacity are affected by the backbone rigidity and ion interactions which control the reorganization and inter-connectivity of the domains [11-13]. The water uptake is characterized by a primary hydration of around $\lambda=6$ (i.e., 6 water molecules per sulfonic acid site), followed by a secondary swelling of the polymer matrix to a $\lambda=14$ (saturated vapor) and $\lambda=22$ (liquid water) [14, 15]. Water uptake has been modeled by various means, mainly using an energy-balance approach wherein the chemical energies related to dissolution are countered by the mechanical energies related to swelling and backbone deformation [16-23]. While these models have shown significant insight into the quasi-equilibrium water content, their use as models of transport have been limited since they inherently neglect the connectivity between the water domains. To complicate matters, it is also known that a PEM exhibits significantly different water uptake and physical properties depending on the phase of water in contact with the surface, namely liquid or vapor [15, 24-26]. Such a change in uptake is thought to perhaps be due to surface morphological changes of the PEM when exposed to the different environments as discussed by Kreuer [14], Freger [26, 27], and explored by Weber and coworkers [24, 28-31]. What is known for certain is that the domain sizes change with liquid versus vapor equilibration as measured by $\mathrm{x}$-ray scattering and macroscopic property measurements [32]. Overall though, the modeling and understanding of transport properties such as ion conductivity are not nearly as developed as that of bulk water uptake.

Various models of proton conduction in PEMs have shown the possible existence of different mechanisms of transport [33-38]. The first is a proton hopping or Grotthuss mechanism wherein the proton moves across a hydrogen-bond network mainly through concerted molecular bond rotations of the hydrated Eigen or Zundel clusters [38-40]. The second mechanism is a vehicular mechanism that is associated with movement of a hydronium ion. The first mechanism is known to be fast and the second slower. As mentioned, there are two transport modes where for the 
liquid-equilibrated transport mode, Grotthuss type conductivity is believed to be dominant, and for vapor-equilibrated it is thought to be more vehicular. The reason for this is that the domain sizes are larger for liquid-equilibration and subsequently there is more of a bulk-like water region $[41,42]$. However, there is undoubtedly a mixture of modes, wherein the connectivity becomes increasingly important, and dominant transport pathways emerge. While the two mechanisms have been explored at the atomic scale through ab-initio and molecular dynamics [42-45], or at the coarse grain scale through dissipative particle dynamics and other methods [46-48], scaling up to a macroscopically measurable quantity across the membrane has been a challenge due to the connectivity issues and disparate length scales of the phenomena; a true, efficient multiscale representation of the conductivity does not exist.

To model the connectivity between hydrophilic domains efficiently and resolve macroscopic transport properties, one can utilize a resistor-network approach similar to pore-network modeling in porous media $[49,50]$. While the approach is not new and demonstrates many of the experimental observations such as a percolation threshold for conduction [51], it is not fully developed for PEMs wherein transport is multiscale and occurs within a dynamic framework with varying morphology and transport properties. The most exemplary of its adoption to PEMs was accomplished by Eikerling et al. [52, 53] and subsequently by Weber et al. [28, 54], wherein such an approach was shown to be able to bridge the gap between the different membrane transport modes (i.e., liquid-equilibrated or vapor-equilibrated) in a physically consistent framework. Eikerling et al. and Weber et al. both utilized a framework of essentially swollen and collapsed channels and used tangential evidence to derive the effective channel-size distributions. Recently, some groups have used a more rigorous approach for modeling transport wherein they accounted for the network structure in more detail [55-58]. There is a still a need to improve the techniques and fidelity of the models, as well as exercise them in terms of exploring and setting design targets. In this paper, we explore conductivity as a function of network topology and ratio of conductivities between the two modes in order to both prove out the approach and help establish design targets and understanding for PEM development. 
The outline of the paper is as follows. First, the modeling methodology is presented. Next, several test cases are explored in terms of conductivity ratio and bulk network dimensionality. Then the modeling of transport more relevant to Nafion is carried out using recently measured values for its channel size and connectivity, where the different conductivity values represent bridging or perhaps defect sites. Finally, a general discussion of the findings is made followed by the summary. 


\section{Modeling}

Two related modeling approaches were used to calculate effective conductivities of ionconducting polymer electrolytes. For the general case of a block co-polymer (BCP), a resistor network was used, which uses a finite-difference scheme to estimate the effective conductivity of the network. For the more specific case of ionomers like Nafion ${ }^{\circledR}$, a pore-network approach was used wherein the conductance values between neighboring nodes is determined from physical considerations of the specific 'pore structure'. Both of these approaches are outlined in more detail in the sections that follow.

\subsection{General Solution Procedure}

For both the BCP and Nafion studies, the effective conductivity of the network was found in the same manner. Using the conductivity $\kappa$ of the conducting material, and the size information for the conduit connecting nodes (or pores) $i$ and $j$, the conductance $g_{i j}$ conduit can be found. The ion flow in the network follows Ohm's law, such that the flow of current $I$ between two neighboring pores $i$ and $j$ is proportional to the voltage difference $\Delta \mathrm{V}$ and the conductance between them as:

$$
I=g_{i j} \Delta V
$$

The current entering and exiting each node will sum to zero for steady-state conditions with no reaction terms. Thus, a current balance around node $i$ gives:

$$
0=\sum_{k=1}^{n} g_{i k}\left(V_{k}-V_{i}\right)
$$

where node $i$ has $n$ neighboring pores. Performing this balance on each node in a network with $N$ nodes results in a linear system of $N$ equations that can be easily solved to find the $V$ in each node. The effective conductivity $S_{E F F}$ of the network can be found by applying voltage boundary conditions on two opposing faces of the network and solving the system of equations resulting from the application of Eq. (2) to every node. With the voltage in each node known, it is possible to calculate the total flow of current through a control surface. The key to the present resistor or pore-network simulations is to find meaningful values for $g_{i k}$ to be used in Eq. (2). 
The modeling domains used here are illustrated in Figure 1 for the $2 \mathrm{D}$ and $3 \mathrm{D}$ cases. The inset views show a close-up of the pore-throat-pore conduit. The pores are represented by cubes and the throat are drawn as lines for clarity, although they were treated as cylinders in the simulations. In all the simulations presented below, the voltage gradient was applied in the $z$ direction.

\subsection{Resistor Network Modeling of Block Co-Polymers}

Resistor values for the BCP network were determined by assigning each node in the network to be either conducting or non-conducting. Once an assignment has been made to each node, the resistance of the total conduit connecting two neighboring nodes $i$ and $j$ was found from

$$
\frac{1}{g_{i j}}=\frac{0.5 L_{C}}{\kappa_{i} A_{C}}+\frac{0.5 L_{C}}{\kappa_{j} A_{C}}=\frac{1}{2 L_{C}}\left(\frac{1}{\kappa_{i}}+\frac{1}{\kappa_{j}}\right)
$$

where $L_{C}$ is the lattice constant of the network, $A_{C}\left(A_{C}=L_{C}{ }^{2}\right.$ since the network is cubic) is the cross-sectional area between nodes, and $\kappa_{i}$ is the conductivity of the phase assigned to node $i$. This is illustrated in Figure 2(a) for three neighboring cells. Cells $1 \& 2$ are of phase A, while cell 3 is of phase B. The conductivity of the pathway between cells $2 \& 3$ is the average of the conductivity of phase A and B. The conductivity between cells $1 \& 2$ is simply that of phase $A$ since the phase is the same in both cells. The actual conductance of a conduit must incorporate the length and cross-sectional area between two nodes, as shown in Eq. (3). The relative frequency of each type of phase can be varied, the intrinsic conductivity of each material, and their spatial arrangement can be manipulated in many ways, as discuss below.

\subsubsection{Spatial Distribution of Phases}

In addition to varying relative proportions and conductivities of the two phases, the resistor network approach allows for easy investigation of the effect of spatial distribution of the two phases. In the base case, conducting and non-conducting elements were dispersed randomly throughout the domain. The impact of spatial correlation between the locations of conducting and non-conducting elements was also investigated. Spatial correlations were imparted by applying a convolution filter to an $\mathrm{N}$-dimension random number field of the same shape as the desired network $\left(N_{x}, N_{y}, N_{z}\right)$, with the structuring element selected to impart correlations of 
varying strength and distance in each spatial direction. The structuring element $S$ was always a $3 \times N_{D}$ array (where $N_{D}$ is the number of dimensions in the model), with entries corresponding to a minimally connected cubic topology (i.e. $2 \times N_{D}$ neighbors). The values in $S$ are described as $\left[A_{x}\right.$, $\left.A_{y}, A_{z}\right]$ meaning that $A_{x}$ was placed at locations $(0,1,1) \&(2,1,1), A_{y}$ was placed at $(1,0,1) \&$ $(1,2,1)$, and $A_{z}$ was placed at $(1,1,0)$ and $(1,1,2)$. This procedure had the side effect of converting an initially random distribution into a normal one. A random number field was re-obtained by applying the inverse cumulated distribution function of the normal distribution using its mean and standard deviation. Some 2D examples are shown in

Figure 3 including a random field, a field with $S=[2,2]$, and with $S=[4,1]$. This random number field can then be converted to a Boolean mask by setting all elements with a value below a certain threshold to phase $A$ and the remainder to $B$, to look at percolation behavior.

The end result of this simulation protocol is a 'site' percolation phenomena $[59,60]$. In site percolation, conduction only occurs between two neighboring pores if they are both conductors. This physically represents the fact that the material surrounding both nodes is conductive and therefore conduction can occur between the two nodes. Conversely, a node filled with nonconducting phase means that none of its neighboring resistors are connected. Referring to Figure 2(a), if phase $B$ is a poor conductor then $R_{2-3}$ will be very high despite the fact that half of the resistor is the higher conductivity $A$ phase. Figure $4(a)$ shows the conductivity behavior of a site-percolation problem as the effective conductivity of the materials versus the volume fraction of the conducting phase. The conductivity of the non-conductor was set to $10^{-5}$ times lower than the conductor, so the final value at low $\phi$ plateaus at this value. A key feature of these percolating systems is the existence of a 'percolation threshold', which is the volume fraction below which the conductivity drops precipitously. This behavior is typically described with an equation of the form:

$$
\kappa_{E F F}=\kappa\left(\phi-\phi_{C}\right)^{\tau}
$$

where $\kappa_{\mathrm{EFF}}$ is the effective conductivity of the network, $\kappa$ is the intrinsic conductivity of the conducting phase, $\phi_{c}$ is the critical volume fraction that indicates the location of the sharp drop in conduction, and $\tau$ is a fitting parameter that is dependent on various aspects of the 
simulation including the dimensionality, connectivity and even the size of the domain.

\subsection{Pore Network Modeling of Nafion}

The pore-network approach to model transport is built upon the concept of a resistor network, but instead of arbitrarily assigning resistor values, they are estimated from geometric and topological knowledge of the pore structure. Nafion does not have a pore network in the traditional sense, where invading and defending phases interact in a network of voids, but there is a clear pore and throat-type structure to the phase-segregated hydrophilic domains.

\subsubsection{Pore and Throat Sizes}

The size of the pore and throats in Nafion have been intensively studied in recent years [13, 14, $22,55,56,61-65]$ including some recent direct visualization of the hydrated structure [66]. The general picture is that pore bodies of mean size around 5 to $10 \mathrm{~nm}$ that are spaced between 12 and $16 \mathrm{~nm}$ apart in dry versus fully humidified membranes, respectively. These pores are connected by throats of 2 to $4 \mathrm{~nm}$, with lengths of a few $\mathrm{nm}$. Figure 2(b) shows the meaning and definitions of these various size parameters in the context of a possible pore network. The pore-size distribution for a saturated-vapor-equilibrated Nafion network was obtained by assigning random numbers to each pore, then using the inverse cumulative distribution function of the Weibull function with an offset as given by:

$$
R=\beta(-\ln (1-p))^{\frac{1}{k}}+b
$$

with $\beta=1.8 \times 10^{-9} \mathrm{~m}, k=2.2$, and $b=1 \times 10^{-8} \mathrm{~m}$. A similar approach was used for throat radii, but the throat seed values were taken as the minimum value in the two adjacent pores. Eq. (5) was then applied with $\beta=1 \times 10^{-10} \mathrm{~m}, k=2.5$, and $b=0 \mathrm{~m}$. The pore- and throat-size distributions are shown in Figure 5. The throat length was found from the difference in lattice spacing and neighboring pore radii.

\subsubsection{Impact of Water Uptake and Swelling}

One of the more challenging aspects of modeling Nafion is that the structure morphs with water uptake, so that at low water contents the pores and throats are contracted, while at higher 
water contents they swell. Incorporating this behavior into the present model was accomplished as follows. A suitable pore- and throat-size distribution was established for a fully hydrated material as outlined in the previous section. In this state, the material consists of about $42 \%$ conducting phase by volume, $\phi$. The value of $\phi$ was related to the membrane water content, $\lambda$ (moles $\mathrm{H}_{2} \mathrm{O}$ per $\mathrm{SO}_{3}{ }^{-}$), using the water and dry-polymer molar volumes [28]:

$$
\phi=\frac{\lambda \cdot 18}{550+\lambda \cdot 18}
$$

where an 1100 equivalent weight $\left(\mathrm{g} /{\mathrm{mole} \mathrm{SO}_{3}}^{-}\right)$Nafion is used. As the material becomes dehydrated, meaning the value of $\lambda$ drops, the polymer shrinks macroscopically by about $20 \%$, and the volume fraction of conducting phase, $\phi$, decreases according to Eq. (6). A new estimate for the pore and throat diameters was found by first shrinking the network spacing to account for the macroscopic size change, then scaling the pore and throat volumes to satisfy Eq. (6). This is schematically illustrated in Figure 2(c). For pores, the new diameter was found directly as the diameter of a sphere with the new contracted volume. For the throats it was a bit more involved. The pores an each end of a throat are shrinking, tending to lengthen the throat; yet the total material contracted by about $20 \%$ from the fully hydrated state to the dry one at the bulk scale thus driving pores closer together. Making these adjustments, the new throat length was determined, and throat diameters were found in a manner similar to the pore bodies, i.e., by assuming the total volume of the cylindrical throat has been reduced in proportion to the decrease in $\phi$. The pore- and throat-size distribution of the network at $\lambda=8$ is shown in Figure 5 , and a strong shift towards smaller pore and throat diameters is seen. An interesting result of this approach is that throat lengths tended to increase as hydration was lowered. This occurred since the volumes and therefore diameters of the pores shrink faster than the bulk material. Although perhaps not entirely physically accurate, the above approach and methodology demonstrate an initial attempt of determining a quasi-equilibrated network for describing transport.

\subsubsection{Position-Dependent Conductivity}

A key feature of the present model was to impart a position-dependent conductivity in the conduits such that the conductivity near the walls was lower than in the bulk regions away from 10 
the walls $[34,35,38]$. This behavior was approximated using the following expression to describe the conductivity $\kappa$ at a distance $r$ from the center of a throat of radius $R$ :

$$
\kappa(r)=\kappa_{w}+\left(\kappa_{B}-\kappa_{W}\right) \frac{1}{1+\mathrm{e}^{C\left(R-r-\delta_{50}\right)}}
$$

where $R$ is the radius of the throat, $\kappa_{B}$ is the conductivity in the bulk region, $\kappa_{W}$ is the conductivity at the wall, $\delta_{50}$ is the half-thickness of the boundary region between the wall and the bulk over which the conductivity varies from $\kappa_{W}$ to $\kappa_{B}$. The coefficient $C$ is a scaling factor which controls the distance from the wall at which the conductivity approaches $99 \%$ of the bulk value, $\delta_{99}$ :

$$
C=\frac{\ln (1 / 0.99-1)}{\delta_{99}-\delta_{50}}
$$

Figure 6 shows a representative conductivity profile for a conduit of radius $R$, a $\delta_{50}$ value of $0.4 R$ and a $\delta_{g g}$ value of $0.6 R$. This function is not easily integrated analytically, so a numerical integration was performed to determine an average conductivity for each throat.

The position dependence of the conductivity means that in very small throats, the entire conduit will be close to a wall. The average conductivity of such a throat would be essentially zero (or $\kappa_{W}$ ), so conduction between the two connected pores will cease. This leads to the second type of percolation known as 'bond percolation' $[50,59,60]$, which applies to transport in porous materials. In the context of traditional pore-network modeling of water invasion into porous materials, a throat can only be filled if both its adjacent pores are filled; while both pores can be filled (via other throats) while their common throat remains unfilled. It differs in subtle but important ways from 'site percolation'. 


\section{Results and Discussion}

\subsection{Dual-Phase Conducting Network}

There are various reasons to examine the issues of conduction in networks wherein there are two phase-segregated domains with different conductivities. As mentioned above, one such example is where two different intrinsic conduction mechanisms exist (e.g., hopping and vehicular mechanisms). While the more conductive phase will dominant, it may be that these are linked only by traversing the lower conduction route at intersections or perhaps where defects exist that no longer allow for the faster conduction mechanism. The developed model is ideal to study this type of percolation and transport. In addition, block copolymers naturally phase segregate into typically conductive and structural elements, where percolation is critical [67], and form various representative elements that can be of various dimensionality from 3-D gyroids, to 2-D lamellae, to 1-D cylinders [68-72]. Similar to the random copolymers, defects in block-copolymer structures can also cause a change of conductivity within the representative element. Therefore, it is worthwhile to explore how the effective conductivity is altered as a function of both the volume fractions of the high and low conducting phases as well as the difference between those values.

Figure 7 shows the variation of total normalized conductivity as a function of the volume fraction of the less-conducting backbone phase for 2 different phase conductivity ratios $(\alpha=$ $\left.\kappa_{\text {Backbone }} / \kappa_{\text {(onductor }}\right)$. Each plot also shows the impact of dimensionality as a parameter. The range of normalized effective conductivity varies between 1 and $\kappa_{\text {Backbone }}$ for all dimensions. The 1-D case shows a much sharper decrease as the amount of less-conductive component is increased than the higher dimensional cases; this behavior is simply due to the fact that protons cannot avoid traversing the low-conductivity regions in a 1-D topology. This situation may seem irrelevant, but one can imagine cases such as lab-on-a-chip, conduction through cylinders, and microfluidics and other MEMS devices where thin linear strips of ionomer may be found. The dimensions of the strip and the size of the phase segregated regions must be considered for the 1-D case. The 2-D and 3-D cases are more relevant to common situations. In-plane conductivity 12 
measurements are commonly used to measure ionic conductivity of polymer films. In particularly thin films the situation may approach 2-D conduction where there is alignment of domains [73-78]. 2-D conduction is somewhat reduced compared to the 3-D case, but not as dramatically as the 1-D materials. The overall message is clear: higher dimensionality means that ions have more options to traverse around obstacles and therefore the effective conductivity is higher. While this conclusion is relatively trivial, the quantification represented by these figures allows them to be used as design metrics for those synthesizing new membrane materials.

It can be quite informative to view the voltage profile and the flux distribution in percolating systems. Figure 8 shows the simulation results of a 2-D system. The conducting phase fraction was 0.50 , and the conducting sites were distributed randomly as shown by the red locations in Figure 8(a). Because this is a site-percolation problem, a resistor is only conducting if both of its neighboring sites are conducting, so the number fraction of high conductivity resistors (red) is lower that the fraction of conducting sites. Figure $8(\mathrm{~b})$ shows the voltage gradient across the network. Some non-uniformities can be seen in this figure owing to the random fluctuations in the conductivity of the underlying network, but the overall gradient shows no unexpected behavior. In Figure 8(c), however, the flux flowing through each resistor shows quite remarkable behavior. It can be clearly seen that the majority of the current is flowing along a few preferred pathways (white and red) while the remainder of the network is unused. This is characteristic of percolating systems since many of the red resistors shown in Figure 8(a) are completely isolated and therefore do not carry current. Interestingly, the fluxes shown in Figure 8(c) bare a striking resemblance to those derived from electrochemical atomic-force microscopy in the literature [79], reinforcing the simulated dominant primary conducting pathways.

In real phase-segregated materials the distribution of the phases is not random, but tends to form aggregates. This behavior can be simulated by distributing the phases with some degree of spatial correlation, as illustrated in

Figure 3. The increased likelihood of finding high conducting nodes near each other impacts the 
percolation behavior. Figure 9 shows some example cases for 3 different degrees of spatial correlation compared to the standard uncorrelated site-percolation result. The values of $\tau$ and $\phi_{C}$ used to fit Eq. (4) are given in the figure legend. The values of $\tau$ and $\phi_{C}$ found here are not rigorous since a large-scale statistical study was not performed, but are demonstrative of important qualitative trends. For more quantitative comparisons, larger domains with more simulations performed near the percolation threshold would be required. It can be seen, however, that generally adding spatial correlation, either with or against the direction of ion flow (which was always in the $z$-direction), increases the effective conductivity and reduces the critical fraction at the percolation threshold. The picture suggests that selectively locating the various phases in a block copolymer or composite structure can change the behavior such that stronger membranes could be produced with similar conductivity simply by tuning the phase segregation through processing, synthesis, or other means.

\subsection{Nafion-Inspired Network}

Conduction in Nafion, as a the prototypical ion-conducting polymer electrolyte, was modeled with a pore-network approach using information derived about the volume fraction of conducting phase and pore and throat sizes to determine the ionic conductance between pores. The measured conductivity of Nafion at room temperature has been correlated using the percolation relationship [28]:

$$
\kappa_{E F F}=0.5(\phi-0.06)^{1.5}
$$

where $\kappa_{E F F}$ has units of $\mathrm{S} / \mathrm{cm}$. This equation is plotted in Figure 10 to provide a baseline against which the various modeling cases can be compared. The first scenario considers only the shrinking of the domain and pore and throat volumes, and applies a constant bulk conductivity value of $\kappa=1.2 \mathrm{~S} / \mathrm{cm}$ throughout. As can be seen in Figure 10, this does not display the characteristic percolation behavior and deviates from Eq. (9) at low values of $\phi$. This is not surprising since the conductivity of the throats is never reduced to negligible values, hence a percolation threshold will not be seen. It is encouraging however, that the simple heuristic algorithm of shrinking the pore and throat volumes captures a fair amount of the observed 14 
decrease in the effective conductivity. The second scenario shown in Figure 10 is the radially variable conductivity model outlined in Section 2.3.3. It was found that bulk conductivity of 1.6 $\mathrm{S} / \mathrm{cm}$ was required, with $1000 \times$ lower at the walls. The transition from the wall to the bulk was set such the $\delta_{50}$ was $0.29 \mathrm{~nm}$ and $\delta_{9 g}$ was $0.30 \mathrm{~nm}$. These values mean that a region of about 0.3 $\mathrm{nm}$ around the wall of every throat had essentially no conductivity. This further implies that any throat which shrinks to a diameter of $0.6 \mathrm{~nm}$ becomes non-conducting, which gives rise to the percolation threshold. Such an approach can be improved using insights from $a b$-initio and molecular calculations, but this is beyond the scope of the current study. The fact that the percolation threshold of around $\phi_{C}=0.06$ was observed is a positive indication that the simple set of rules used to adjust the morphology with decreasing water content $\lambda$ are capturing the real phenomena. The accepted value for $\phi_{c}$ in bond percolation on cubic lattices is much higher at $0.2488[59,60]$, so the rather low value seen here is not a trivial result. As a final case, it was explored whether this percolation behavior could be obtained with an even simpler model; specifically, all throats of a diameter less than $1 \mathrm{~nm}$ are set to non-conducting. At large $\phi$ values, most throats are larger than this, as can be seen in Figure 5; however, as $\phi$ is decreased, more throats satisfy this criteria leading to the percolation behavior shown. Threshold throat-radius values higher than $1 \mathrm{~nm}$ cause the shift of $\phi_{C}$ to higher values and vice-versa. This value of $1 \mathrm{~nm}$ is fairly close to the $0.6 \mathrm{~nm}$ diameter found in the case with the radially varying conductivity. The bulk conductivity in this case was set to $\kappa_{B}=1.2 \mathrm{~S} / \mathrm{cm}$, as in the first case. It is noteworthy that in the second scenario, it was necessary to increase the bulk conductivity to $\kappa_{B}=1.6 \mathrm{~S} / \mathrm{cm}$ in order to compensate for the fact that the outer shell of each throat was non-conducting.

Finally, it is of interest to examine possible proton conduction through interfacial structures or Nafion thin-films that exist within PEFC catalyst layers, where these thin films form a 3D network throughout the layer in which protons must travel [80]. These thin-films demonstrate reduced conductivity and water uptake [76-78, 81-88] and can limit PEFC performance at low catalyst loadings as discussed recently [89], and the modeling of such transport in thin-films is of active interest [1]. It is well known that when a polymer is confined to thicknesses comparable to its characteristic domain size, its properties and morphology differ from the analogous bulk 15 
materials [90]. To explore the thin-film behavior, it is a simple matter to adjust the domain size in the present simulations to look at the impact of film thickness in terms of percolation, using a domain similar to that shown in Figure 1(a). Figure 11 shows percolation curves of Nafion thinfilms of various thickness, compared to the baseline conductivity for the bulk material given by Eq. (9). The first thing to note is that the case of constant intrinsic conductivity is not impacted at all by the film thickness. In this case, the throat conductance does not transition to a nonconducting state as the throats become smaller, and hence there is no percolation limitation. A more physically relevant situation is that of the case considered above where all throats smaller than $1 \mathrm{~nm}$ in diameter are set to non-conducting. The impact this has on 2-D or thin-film simulations is shown in Figure 11 for domains 1, 2, and 3 layers thick. Recalling that the lattice spacing used in these simulations was $16 \mathrm{~nm}$, this corresponds to 16, 32, and $48 \mathrm{~nm}$ thick films. As can be seen, the $16 \mathrm{~nm}$ film has a much lower conductivity than the 3-D bulk case, and the critical volume fraction is about 2 times higher. It has been observed that in-plane conductivity in thin films does indeed decrease $[75,76,82]$, and the deviation from the 3-D case worsens at lower $\phi$ values. As 2 and 3 layers of nodes are used in the simulations, the effective conductivity behavior quickly approaches that of the 3-D case. This is also in qualitative agreement with the existing experimental observations [76-78, 81-88], which show increased conductivity with thickness that reaches almost complete bulk-like behavior for samples $100 \mathrm{~nm}$ or so thick.

The modeling approach presented herein provides useful insights into the behavior of Nafion and its swelling-dependent behavior, but the model is clearly overly simplistic. The list of possible improvements is virtually endless, and includes features like adding spatial correlation, shrinking large pores faster than small ones, increasing the connectivity of the network, improving the radial conductivity distribution model, and so on. Most of these will improve or alter the quantitative results but will not affect the qualitative picture. The one simplification that was made which could have an important impact on the results was the assumption of a constant intrinsic ionic conductivity inside the conduits as $\lambda$ changed. For instance, it would be possible to reproduce the percolation behavior seen in Figure 10 just by adjusting $\kappa_{B}$, rather than by collapsing pores less than $1 \mathrm{~nm}$ in diameter. Clearly the hydration level of the 16 
membrane will affect the mobility and concentration of ions, so the assumption of a nonvarying intrinsic conductivity in the region far away from the wall at all values of $\lambda$ should be considered in more detail in future work. 


\section{Conclusions}

Resistor- and pore-network modeling methodologies were applied to study ion-conduction in solid polymer-electrolytes. Simple cases of dual-phase conductivities in various dimensions and with different high/low conductivity ratios were considered to illustrate the impact of the relative proportion and intrinsic conductivity of the two constituent materials on the effective conductivity of the polymer. The physics of these simulations resulted in a site percolation behavior. Several case studies were performed: It was demonstrated that 1-D conduction is dramatically impacted by low-conductivity inclusions, which could be of relevance for conduction through cylindrical pores with defects or lab-on-a-chip type applications where thin channels of ionomer may be used. 2-D and 3-D conduction were much less sensitive to nonconducting or low-conducting inclusions as the higher dimensionality allows for ions to flow around blockages. Ion flow was visualized to demonstrate the impact of percolation effects on the streamlines, and it was seen that a small fraction of the total material carries the large majority of the current, which could have implications for new ionomer design. The role of spatial correlation at the mesoscale was briefly investigated revealing that any correlations whether with or against the direction of current flow improved the effective conductivity noticeably. This behavior was attributed to the fact that neighboring nodes are more likely to be high conductors, therefore longer conducting pathways were present. Once the basic behavior of percolating polymer systems was established, attention was turned to the special case of Nafion ionomer which has intriguing water-sorption behavior and both meso and nanoscale spatial dependences for conduction. The conductivity of water-vapor-equilibrated Nafion was modeled using a water-content-dependent pore and throat network where swelling was considered, and with a spatially varying conductivity within the nanodomains. This resulted in very good agreement with experimental trends over the range of water contents from 14 down to 1 . Finally, the model was used to study the impact of thin films on the overall conductivity, and it was found that a film of 1 to 2 nodes (corresponding to 15 to $30 \mathrm{~nm}$ ) thick had much lower in-plane conductivity than the bulk materials. Overall, the pore-network simulation and methodology provided useful insight into the behavior of Nafion, and demonstrated that such 
an approach is a viable way to simulate efficiently the transport properties from the nanoscale through the mesoscale to macroscopically measureable properties. 


\section{Acknowledgements}

This work was supported by the Assistant Secretary for Energy Efficiency and Renewable Energy,

Fuel Cell Technologies Office, of the U.S. Department of Energy under contract number DEAC02-05CH11231 and through a subcontract to McGill University. 


\section{References}

[1] A.Z. Weber, R.L. Borup, R.M. Darling, P.K. Das, T.J. Dursch, W. Gu, D. Harvey, A. Kusoglu, S. Litster, M.M. Mench, R. Mukundan, J.P. Owejan, J.G. Pharoah, M. Secanell, I.V. Zenyuk, Journal of the Electrochemical Society, 161 (2014) F1254-F1299.

[2] A.Z. Weber, M.M. Mench, J.P. Meyers, P.N. Ross, J.T. Gostick, Q.H. Liu, J. Appl. Electrochem., 41 (2011) 1137-1164.

[3] M.S. Dresselhaus, G.W. Crabtree, M.V. Buchanan, Mrs Bull, 30 (2005) 518-524.

[4] http://www.sc.doe.gov/bes/hydrogen.pdf.

[5] M.A. Hickner, B.S. Pivovar, Fuel Cells, 5 (2005) 213-229.

[6] S.J. Hamrock, M.A. Yandrasits, Polymer Reviews, 46 (2006) 219-244.

[7] J.M. Spurgeon, M.G. Walter, J. Zhou, P.A. Kohl, N.S. Lewis, Energy \& Environmental Science, 4 (2011) 1772-1780.

[8] BESAC, DOE, in, 2012.

[9] F.A. De Bruijn, R.C. Makkus, R.K.A.M. Mallant, G.J.M. Janssen, Materials for State-of-theart PEM Fuel Cells, and their Suitability for Operation above $100^{\circ} \mathrm{C}$, in: T.S. Zhao, K.-D. Kreuer, T.V. Nguyen (Eds.) Advances in Fuel Cells, Vol. 1, Elsevier, Amsterdam, 2007.

[10] K.A. Mauritz, R.B. Moore, Chemical Reviews, 104 (2004) 4535-4585.

[11] M. Lebret, B.H. Zimm, Biopolymers, 23 (1984) 287-312.

[12] R.G. Winkler, M. Gold, P. Reineker, Phys. Rev. Lett., 80 (1998) 3731-3734.

[13] A. Kusoglu, S. Savagatrup, K.T. Clark, A.Z. Weber, Macromolecules, 45 (2012) 7467-7476.

[14] K.-D. Kreuer, Solid State Ionics, (2013).

[15] T.A. Zawodzinski, C. Derouin, S. Radzinski, R.J. Sherman, V.T. Smith, T.E. Springer, S. Gottesfeld, J. Electrochem. Soc., 140 (1993) 1041-1047.

[16] V. Freger, Polymer, 43 (2002) 71-76.

[17] V. Freger, J. Phys. Chem. B, 113 (2009) 24-36.

[18] K.D. Kreuer, Solid State Ionics, 252 (2013) 93-101.

[19] K.D. Kreuer, G. Portale, Advanced Functional Materials, 23 (2013) 5390-5397.

[20] A. Kusoglu, B.L. Kienitz, A.Z. Weber, J. Electrochem. Soc., 158 (2011) B1504-B1514.

[21] A. Kusoglu, A.Z. Weber, M.A. Hickner, Chemical Reviews, (2014) in preparation.

[22] A. Kusoglu, A.Z. Weber, Water Transport and Sorption in Nafion Membrane, in: Polymers

for Energy Storage and Delivery: Polyelectrolytes for Batteries and Fuel Cells, American Chemical Society, 2012, pp. 175-199.

[23] A. Kusoglu, S. Savagatrup, K.T. Clark, A.Z. Weber, Macromolecules, 45 (2012) 7467-7476.

[24] G.S. Hwang, D.Y. Parkinson, A. Kusoglu, A.A. MacDowell, Adam Z. Weber, ACS Macro Letters, 2 (2013) 288-291.

[25] D.S. Hussey, D. Spernjak, A.Z. Weber, R. Mukundan, J. Fairweather, E.L. Brosha, J. Davey, J.S. Spendelow, D.L. Jacobson, R.L. Borup, J. Appl. Phys., 112 (2012).

[26] M. Bass, V. Freger, Polymer, 49 (2008) 497-506.

[27] M. Bass, A. Berman, A. Singh, O. Konovalov, V. Freger, J. Phys. Chem. B, 114 (2010) 3784-3790.

[28] A.Z. Weber, J. Newman, J. Electrochem. Soc., 151 (2004) 311-325.

[29] Q. He, I.T. Lucas, A. Kusoglu, A.Z. Weber, R. Kostecki, J. Phys. Chem. B, 115 (2011) 11650-11657

[30] A. Kusoglu, M.A. Modestino, A. Hexemer, R.A. Segalman, A.Z. Weber, ACS Macro Letters, 
1 (2012) 33-36.

[31] B. Kienitz, H. Yamada, N. Nonoyama, A.Z. Weber, Journal of Fuel Cell Science and Technology, 8 (2011) 011013.

[32] G. Gebel, O. Diat, Fuel Cells, 5 (2005) 261-276.

[33] K.D. Kreuer, Solid State Ionics, 136 (2000) 149-160.

[34] K.D. Kreuer, S.J. Paddison, E. Spohr, M. Schuster, Chemical Reviews, 104 (2004) 46374678.

[35] S.J. Paddison, R. Paul, T.A. Zawodzinski, J. Electrochem. Soc., 147 (2000) 617-626.

[36] J. Lobaugh, G.A. Voth, J. Chem. Phys., 104 (1996) 2056-2069.

[37] T.J.F. Day, U.W. Schmitt, G.A. Voth, J Am Chem Soc, 122 (2000) 12027-12028.

[38] M.K. Petersen, G.A. Voth, Journal of Physical Chemistry B, 110 (2006) 18594-18600.

[39] K.D. Kreuer, Solid State Ionics, 94 (1997) 55-62.

[40] N. Agmon, Chem. Phys. Lett., 244 (1995) 456-462.

[41] K.-D. Kreuer, S.J. Paddison, E. Spohr, M. Schuster, Chemical Reviews, 104 (2004) 46374678.

[42] S.J. Paddison, Annu Rev Mater Res, 33 (2003) 289-319.

[43] M.K. Petersen, A.J. Hatt, G.A. Voth, Journal of Physical Chemistry B, 112 (2008) 77547761.

[44] M.K. Petersen, F. Wang, N.P. Blake, H. Metiu, G.A. Voth, Journal of Physical Chemistry B, 109 (2005) 3727-3730.

[45] K. Malek, M. Eikerling, Q. Wang, Z. Liu, S. Otsuka, K. Akizuki, M. Abe, J. Chem. Phys., 129 (2008) 204702/204701-204702/204710.

[46] N. Gavish, J. Jones, Z.F. Xu, A. Christlieb, K. Promislow, Polymers-Basel, 4 (2012) 630655.

[47] C. Wang, S.J. Paddison, Soft Matter, 10 (2014) 819-830.

[48] M.E. Selvan, D.J. Keffer, S.T. Cui, S.J. Paddison, J Phys Chem C, 114 (2010) 11965-11976.

[49] J. Bear, Dynamics of Fluids in Porous Media, Dover Publications, Inc., New York, 1988.

[50] F.A.L. Dullien, Porous Media: Fluid Transport and Pore Structure, 2nd ed., Academic Press, Inc., New York, 1992.

[51] W.Y. Hsu, J.R. Barkley, P. Meakin, Macromolecules, 13 (1980) 198-200.

[52] M. Eikerling, A.A. Kornyshev, A.M. Kuznetsov, J. Ulstrup, S. Walbran, J. Phys. Chem. B, 105 (2001) 3646-3662.

[53] M. Eikerling, A.A. Kornyshev, U. Stimming, J. Phys. Chem. B, 101 (1997) 10807-10820.

[54] A.Z. Weber, J. Newman, J. Electrochem. Soc., 150 (2003) A1008-A1015.

[55] G.S. Hwang, M. Kaviany, J.T. Gostick, B. Kienitz, A.Z. Weber, Polymer, 52 (2011) 25842593.

[56] M.H. Eikerling, P. Berg, Soft Matter, 7 (2011) 5976-5990.

[57] P. Berg, B.E. Benjaminsen, Electrochim Acta, 120 (2014) 429-438.

[58] P. Costamagna, S. Grosso, R.D. Felice, Journal of Power Sources, 178 (2008) 537-546.

[59] B. Bollobás, O. Riordan, Percolation, Cambridge University Press, Cambridge, 2006.

[60] M.B. Isichenko, Rev. Mod. Phys., 64 (1992) 961-1043.

[61] G.M. Divoux, K.A. Finlay, J.K. Park, J.-M. Song, B. Yan, M. Zhang, D.A. Dillard, R.B. Moore, ECS Transactions, 41 (2011) 87-100.

[62] K. Schmidt-Rohr, Q. Chen, Nature Materials, 7 (2008) 75-83.

[63] J. Divisek, M. Eikerling, V. Mazin, H. Schmitz, U. Stimming, Y.M. Volfkovich, J. Electrochem. Soc., 145 (1998) 2677-2683. 
[64] L. Rubatat, A.L. Rollet, G. Gebel, O. Diat, Macromolecules, 35 (2002) 4050-4055.

[65] A.L. Rollet, O. Diat, G. Gebel, J. Phys. Chem. B, 106 (2002) 3033-3036.

[66] F.I. Allen, L.R. Comolli, A. Kusoglu, M.A. Modestino, A.M. Minor, A.Z. Weber, ACS Macro Letters, doi: 10.1021/mz500606h.

[67] W.Y. Hsu, M.R. Giri, R.M. Ikeda, Macromolecules, 15 (1982) 1210-1212.

[68] R.A. Segalman, Materials Science \& Engineering R-Reports, 48 (2005) 191-226.

[69] R.A. Segalman, Science, 321 (2008) 919-920.

[70] I.W. Hamley, The Physics of Block Copolymers, Oxford University Press, New York, 1998.

[71] F.S. Bates, G.H. Fredrickson, Physics Today, 52 (1999) 32-38.

[72] J.K. Kim, J.I. Lee, D.H. Lee, Macromolecular Research, 16 (2008) 267-292.

[73] K.A. Page, A. Kusoglu, C.M. Stafford, S. Kim, R.J. Kline, A.Z. Weber, Nano letters, 14 (2014) 2299-2304.

[74] A. Kusoglu, D. Kushner, D.K. Paul, K. Karan, M.A. Hickner, A.Z. Weber, Advanced Functional Materials, 24 (2014) 4763-4774.

[75] D.K. Paul, K. Karan, J Phys Chem C, 118 (2014) 1828-1835.

[76] M.A. Modestino, D.K. Paul, S. Dishari, S.A. Petrina, F.I. Allen, M.A. Hickner, K. Karan, R.A. Segalman, A.Z. Weber, Macromolecules, 46 (2013) 867-873.

[77] S.K. Dishari, M.A. Hickner, Macromolecules, 46 (2013) 413-421.

[78] S.A. Eastman, S. Kim, K.A. Page, B.W. Rowe, S. Kang, C.L. Soles, K.G. Yager, Macromolecules, 45 (2012) 7920-7930.

[79] E. Aleksandrova, R. Hiesgen, K.A. Friedrich, Physical Chemistry Chemical Physics, 9 (2007) 2735-2743.

[80] M. Lopez-Haro, L. Guetaz, T. Printemps, A. Morin, S. Escribano, P.H. Jouneau, P. BayleGuillemaud, F. Chandezon, G. Gebel, Nat Commun, 5 (2014).

[81] H. Iden, K. Sato, A. Ohma, K. Shinohara, Journal of the Electrochemical Society, 158 (2011) B987-B994.

[82] D.K. Paul, K. Karan, A. Docoslis, J.B. Giorgi, J. Pearce, Macromolecules, 46 (2013) 34613475.

[83] P. Krtil, A. Trojanek, Z. Samec, Journal of Physical Chemistry B, 105 (2001) 7979-7983.

[84] S.K. Dishari, M.A. Hickner, ACS Macro Letters, 1 (2012) 291-295.

[85] P. Bertoncello, I. Ciani, F. Li, P.R. Unwin, Langmuir, 22 (2006) 10380-10388.

[86] Z. Siroma, R. Kakitsubo, N. Fujiwara, T. Ioroi, S.I. Yamazaki, K. Yasuda, Journal of Power Sources, 189 (2009) 994-998.

[87] A. Kongkanand, The Journal of Physical Chemistry C, 115 (2011) 11318-11325.

[88] A. Kusoglu, D. Kushner, D.K. Paul, K. Karan, M.A. Hickner, A.Z. Weber, Advanced Functional Materials, (2014) 4763-4774.

[89] A.Z. Weber, A. Kusoglu, Journal of Materials Chemistry A, 2 (2014) 17207-17211.

[90] T.P. Russell, P. Lambooy, G.J. Kellogg, A.M. Mayes, Physica B, 213 (1995) 22-25. 


\section{Captions}

Figure 1: $\quad$ Views of the modeled domain for 2D (left) and 3D (right) simulations, colored according to the applied voltage gradient which was always in the $z$-direction. The cubes indicate the pore bodies and the white mesh indicates the throat network.

Figure 2: $\quad$ Schematic diagram illustrating the key dimension used to determine resistornetwork properties.

Figure 3: Spatial distribution of ionic conductance including random, isotropically correlated $(2,2)$ and anisotropically correlated $(4,1)$. All three images possess a random distribution of values between 0 and 1 . Red represent random number near 1 and dark blue is numbers near 0.

Figure 4: $\quad$ Typical percolation curve for conduction through a random 3D network with no spatial correlations. Site percolation is shown on the left, and bond percolation on the right. The markers are simulated data points and the line represent Eq. (4) with the coefficients shown in each figure. The critical volume fractions are taken from the accepted values for these problems, while $\tau$ was determined by fitting.

Figure 5: $\quad$ Pore- and throat-size distributions for fully humidified Nafion at $\lambda=14$ (blue), 
and $\lambda=8$ (green).

Figure 6: $\quad$ The normalized radial conductivity profile (blue line) assumed to exist in the conducting conduits in the ionomer as described by

Figure 7: Effective conductivity of the ionomer as a function on volume fraction of mechanically supportive (ionically insulating) phase for two different $\alpha$ values. Each graph shows the results of 1D (green), 2D (yellow) and 3D (red) systems.

Figure 8: $\quad$ lon transport simulations in 2-D random geometry. Left: Distribution of high (red) and low (blue) conductance values. Middle: Voltage field across network from high (red) on top to low (blue) on the bottom. Right: Ion flux lines through the network showing a few limited pathways (red to white) carrying most of the current.

Figure 9: Influence of spatial correlations on the effective conductivity, as illustrated by percolation curves for 3 cases with different structuring elements used to impart spatial correlation.

Figure 10: Nafion conductivity as a function of $\phi$ for various scenarios of throat conductivity.

Figure 11: In-plane conductivity of Nafion thin films, with thickness indicated by the number of layers in the direction perpendicular to the current flow. Each layer of nodes 
$\mathrm{nm}$. 


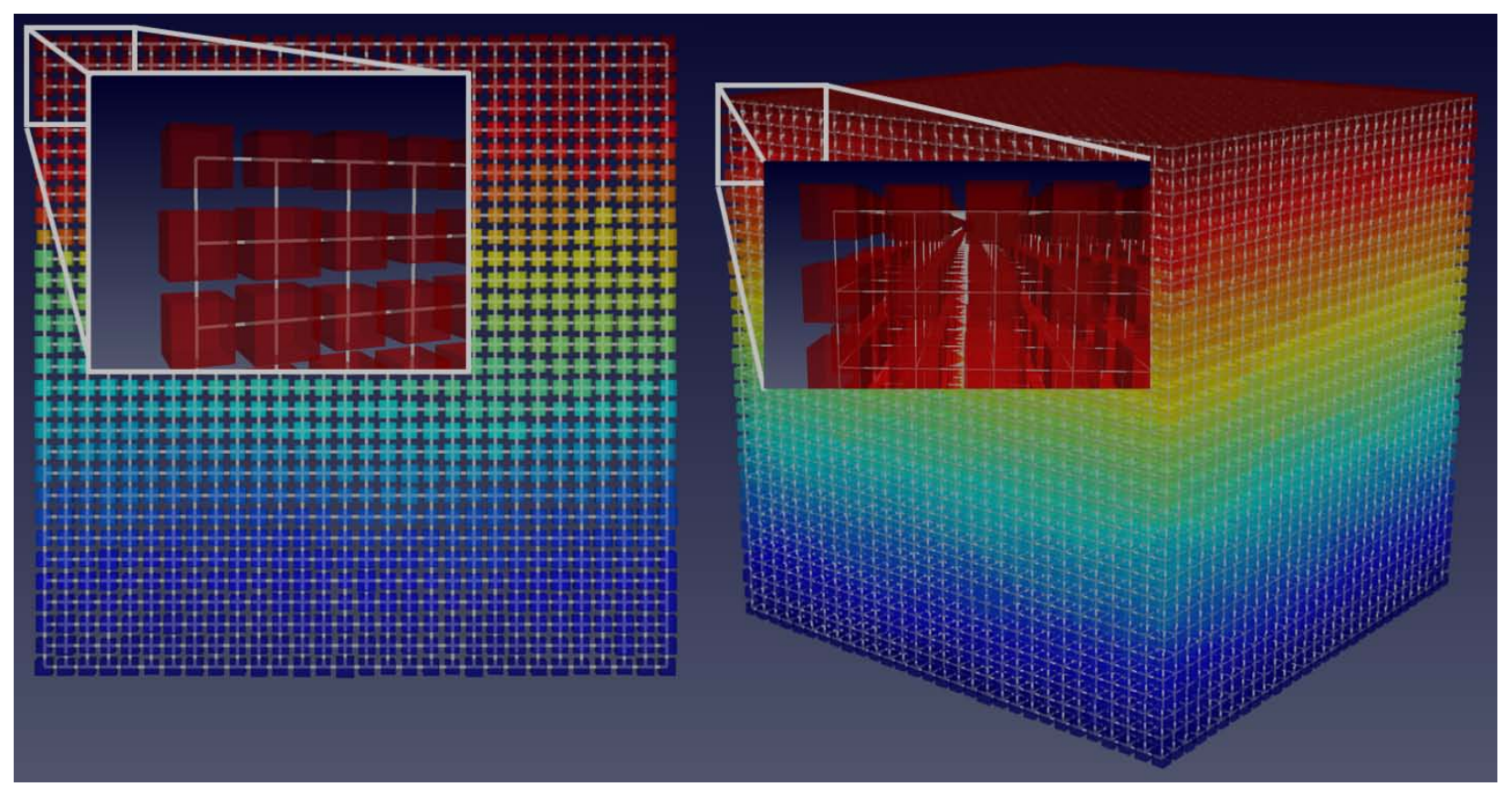

Figure 1: Views of the modeled domain for 2D (left) and 3D (right) simulations, colored according to the applied voltage gradient which was always in the z-direction. The cubes indicate the pore bodies and the white mesh indicates the throat network. 


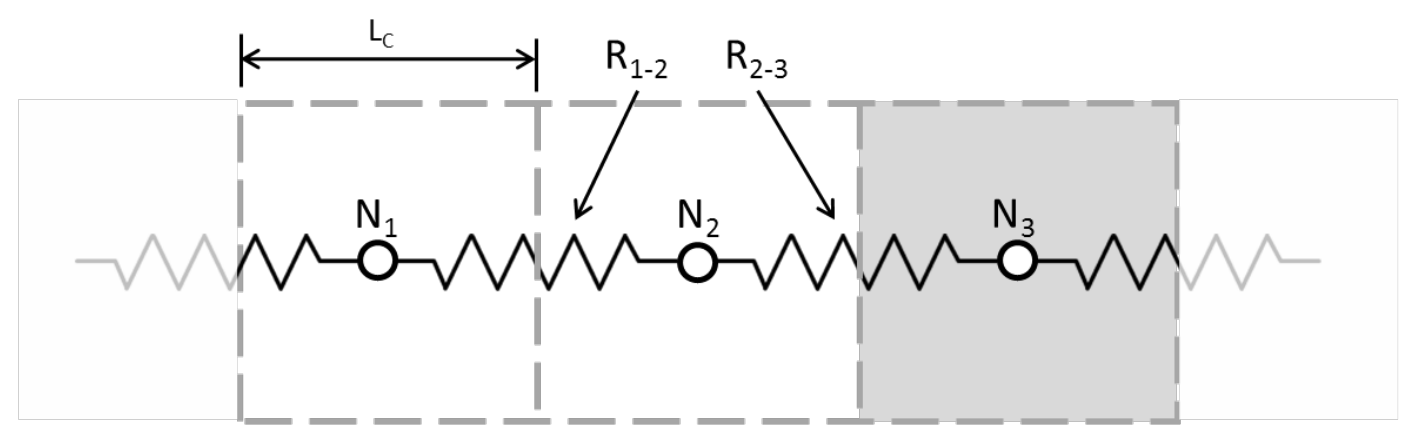

(a)

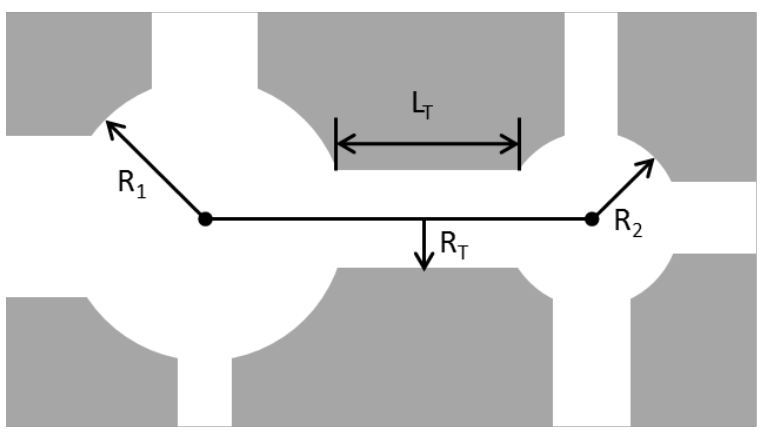

(b)

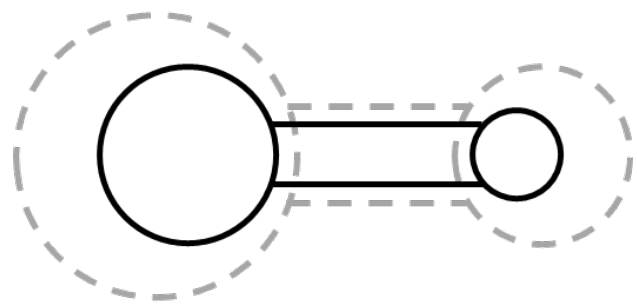

(c)

Figure 2: Schematic diagram illustrating the key dimension used to determine resistor-network properties. (a) The resistance values between pores includes contributions from both pores which it connects. (b) For the Nafion simulation pores were modeled as spheres with throat connections as cylinders. (c) Upon dehydration the volumes of pores and throats shrink, paradoxically leading to a lengthening of the throat. 


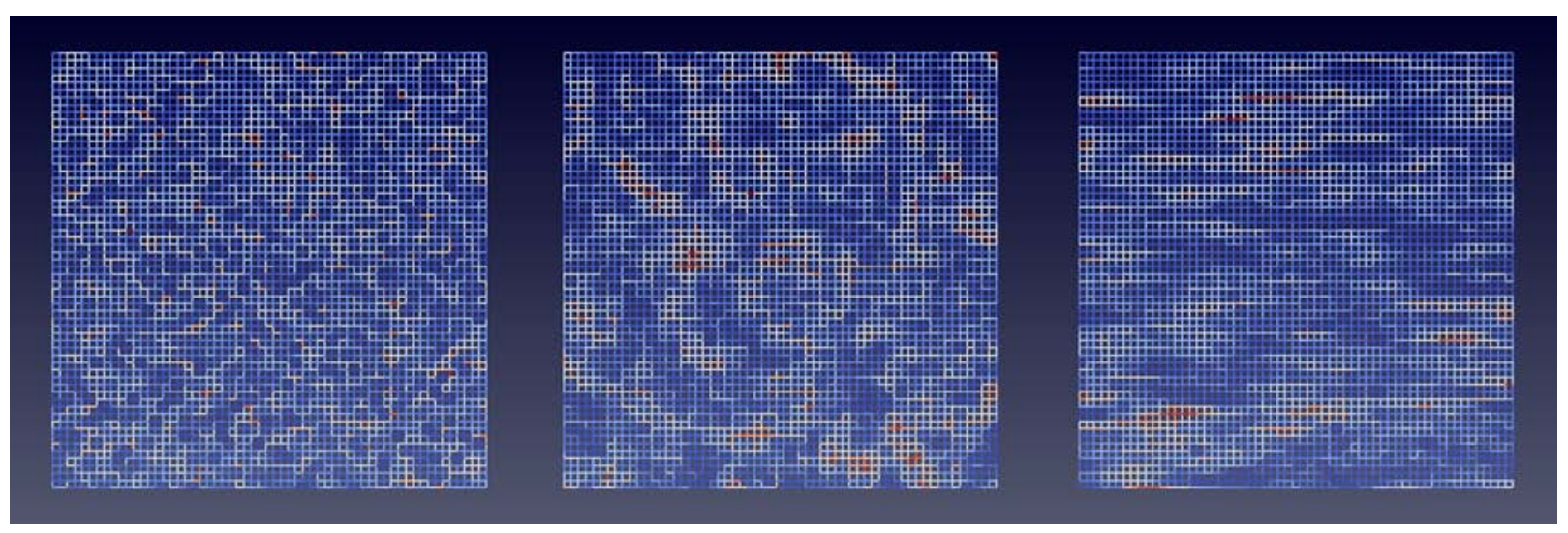

Figure 3: Spatial distribution of ionic conductance including random, isotropically correlated $(2,2)$ and anisotropically correlated $(4,1)$. All three images possess a random distribution of values between 0 and 1 . Red represent random number near 1 and dark blue is numbers near 0 . 

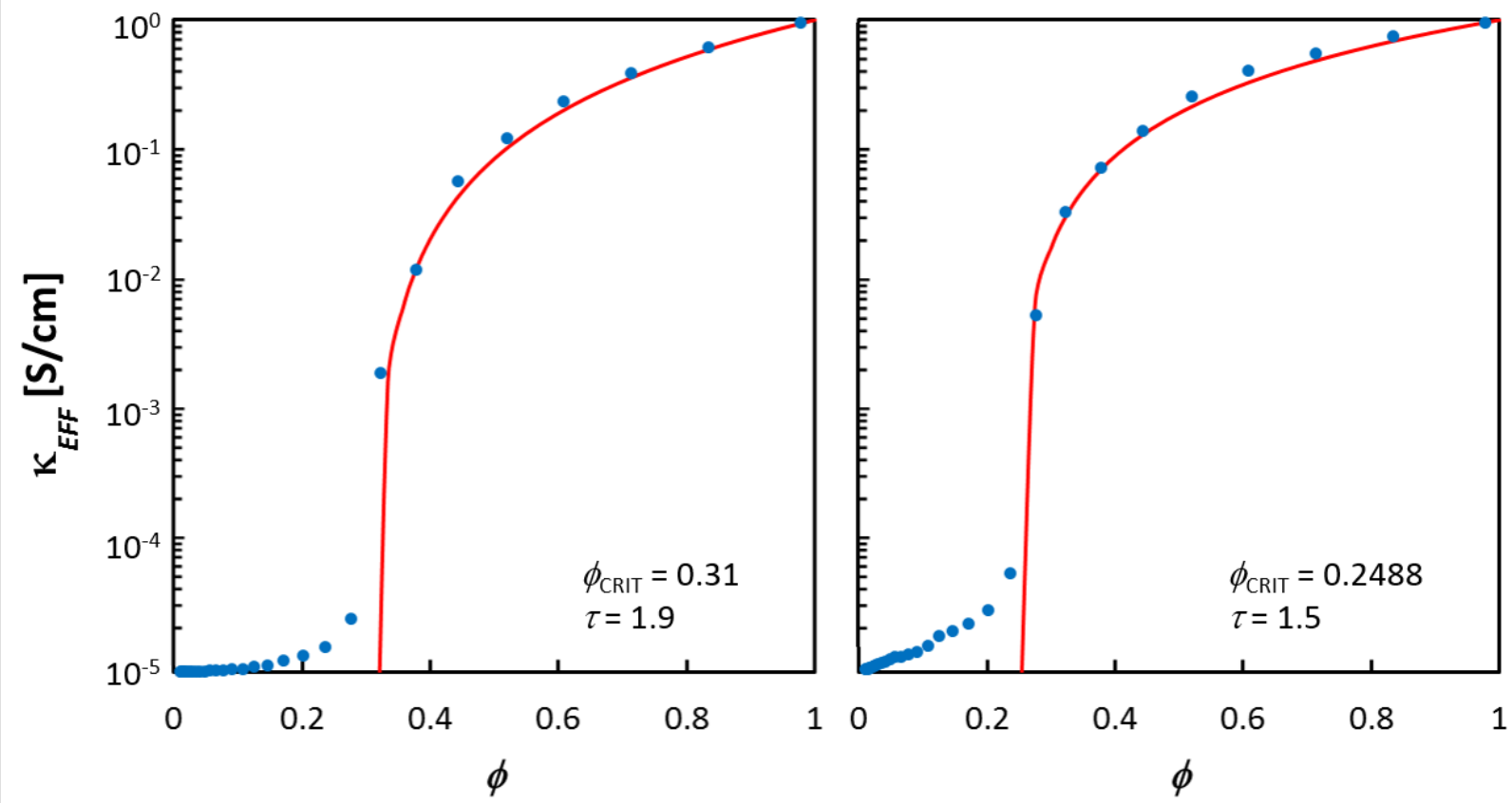

Figure 4: Typical percolation curve for conduction through a random 3D network with no spatial correlations. Site percolation is shown on the left, and bond percolation on the right. The markers are simulated data points and the line represent Eq. (4) with the coefficients shown in each figure. The critical volume fractions are taken from the accepted values for these problems, while $\tau$ was determined by fitting. 

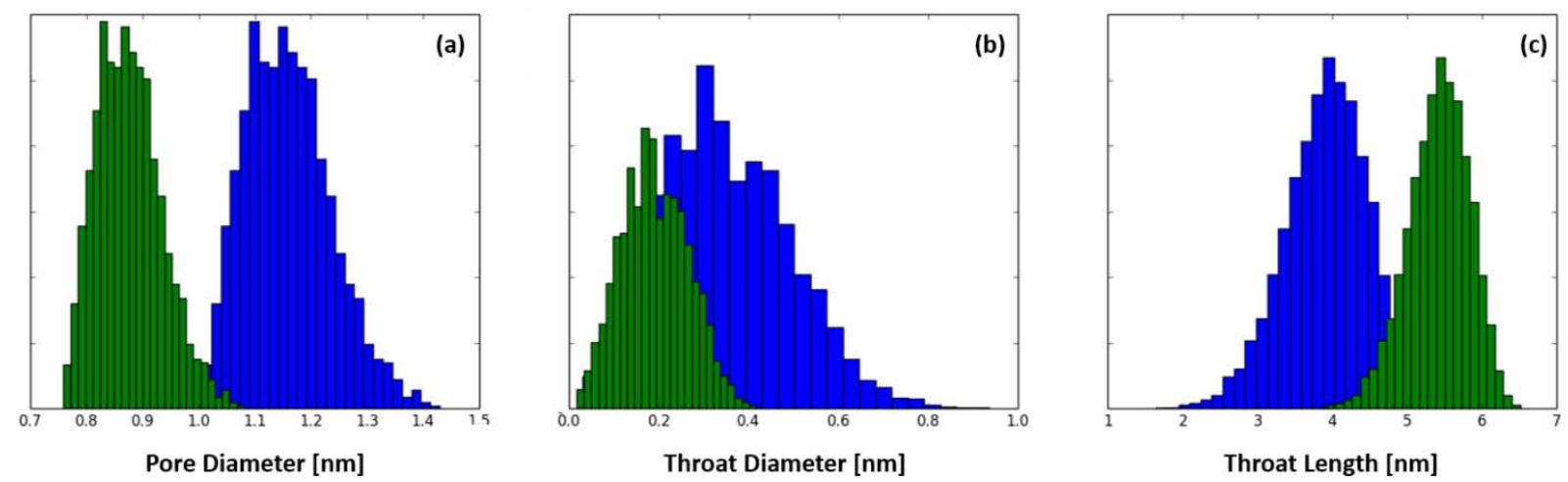

Figure 5: Pore- and throat-size distributions for fully humidified Nafion at $\lambda=14$ (blue), and $\lambda=8$ (green). 


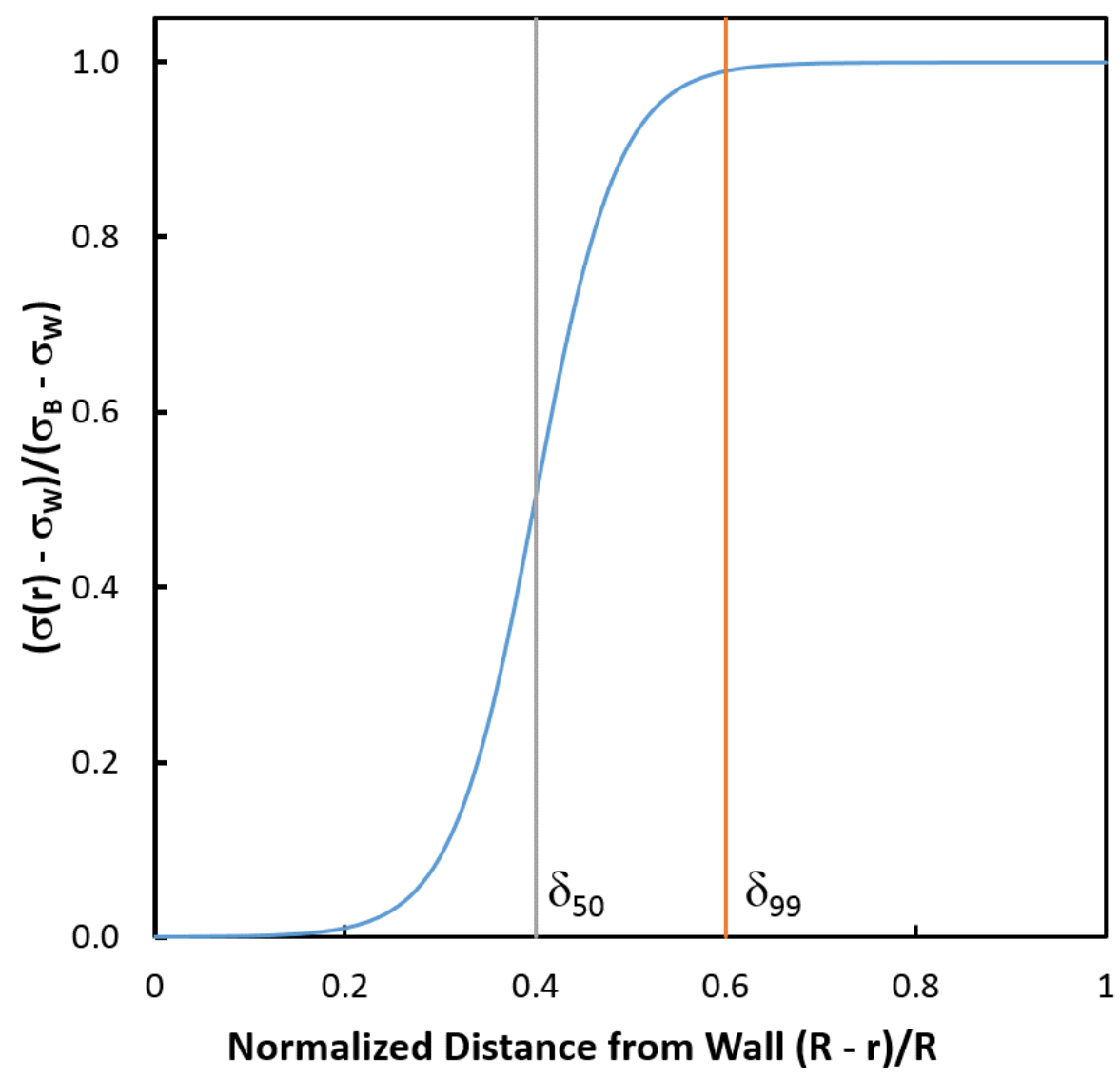

Figure 6: The normalized radial conductivity profile (blue line) assumed to exist in the conducting conduits in the ionomer as described by Eq. (7). The grey and red lines indicate the normalized radial positions at which the conductivity reaches $50 \%$ and $99 \%$ respectively, which are adjustable parameters in Eq. (7). 

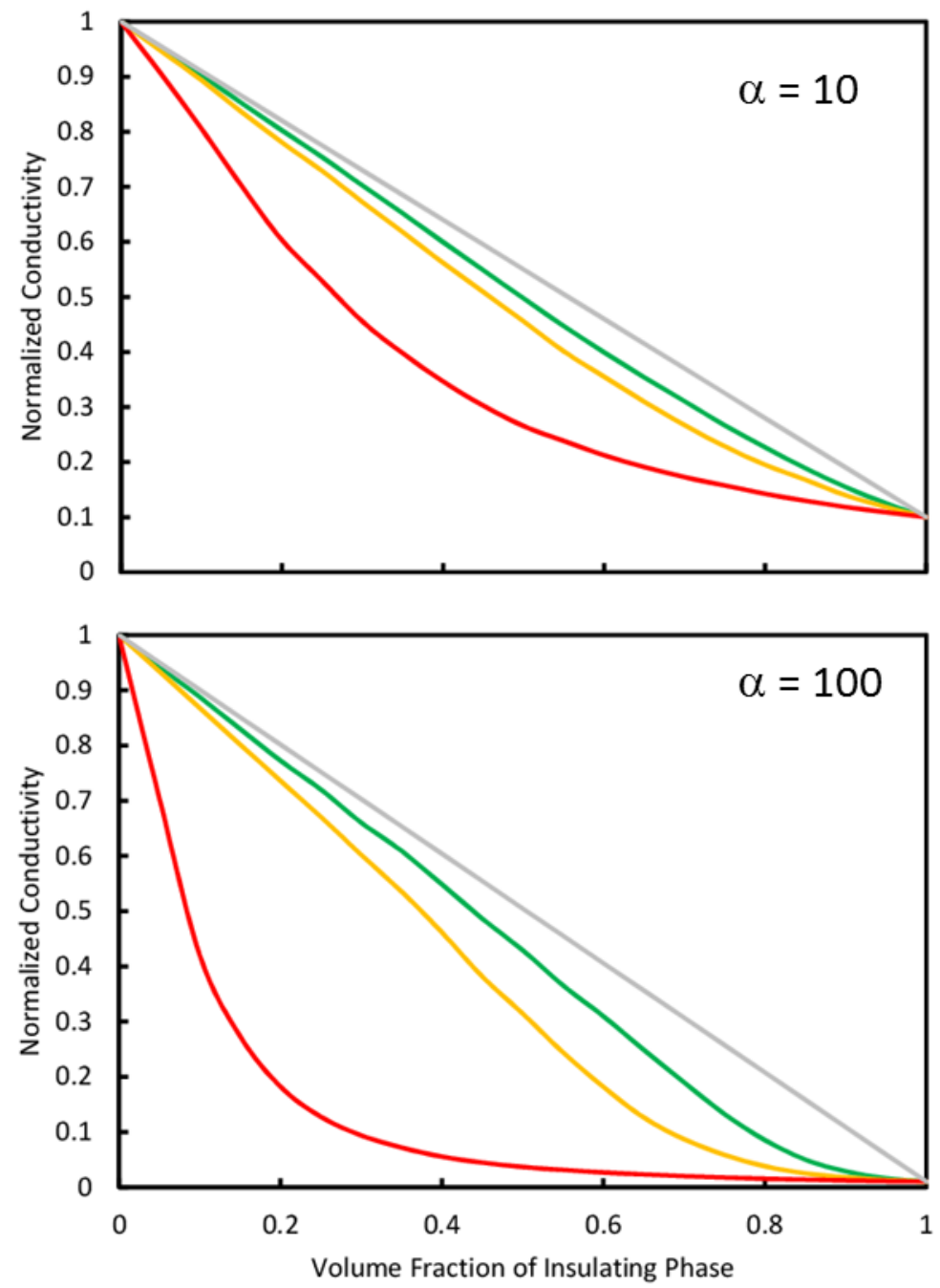

Figure 7: Effective conductivity of the ionomer as a function on volume fraction of mechanically supportive (ionically insulating) phase for two different $\alpha$ values. Each graph shows the results of 1D (green), 2D (yellow) and 3D (red) systems. 


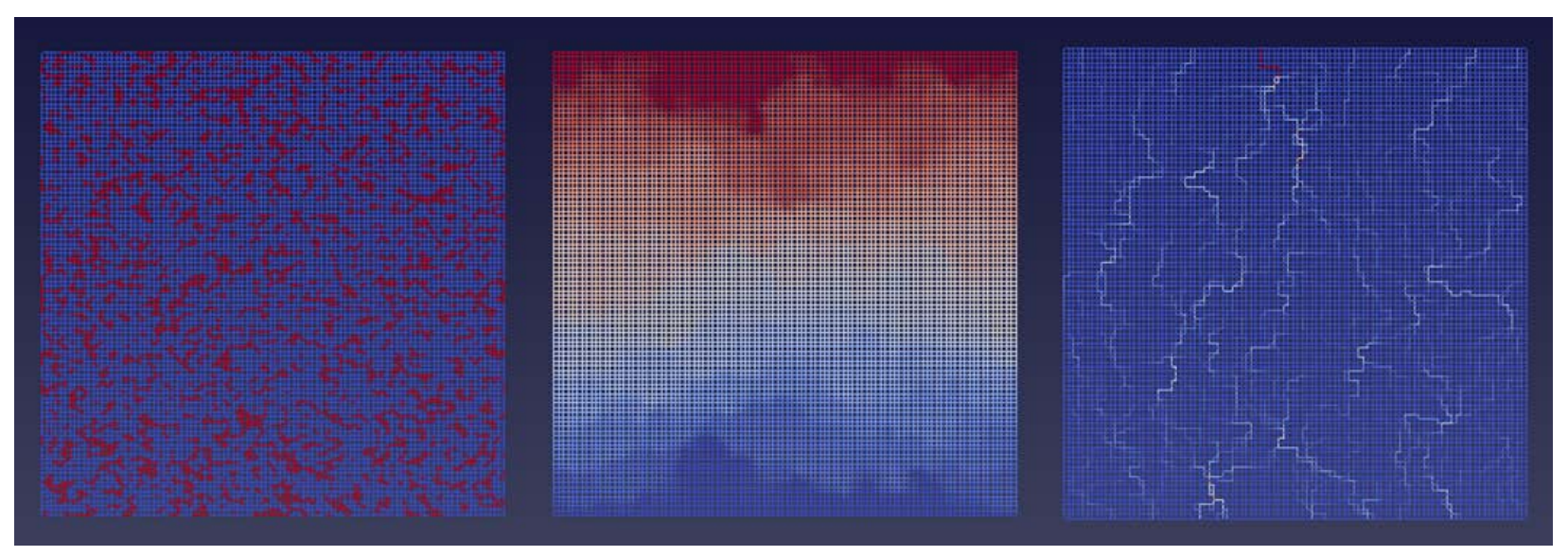

Figure 8: Ion transport simulations in 2-D random geometry. Left: Distribution of high (red) and low (blue) conductance values. Middle: Voltage field across network from high (red) on top to low (blue) on the bottom. Right: Ion flux lines through the network showing a few limited pathways (red to white) carrying most of the current. 


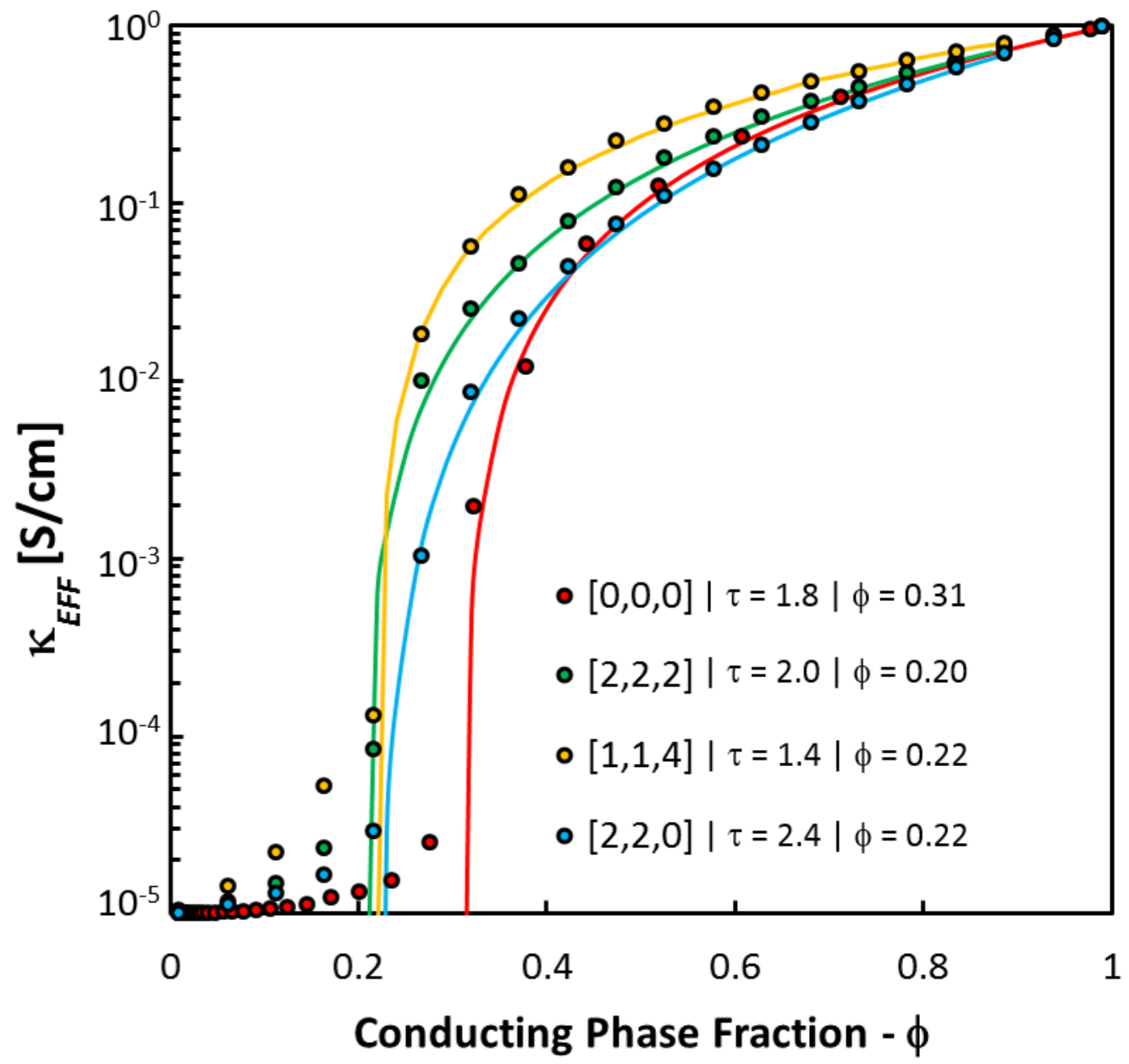

Figure 9: Influence of spatial correlations on the effective conductivity, as illustrated by percolation curves for 3 cases with different structuring elements used to impart spatial correlation. 


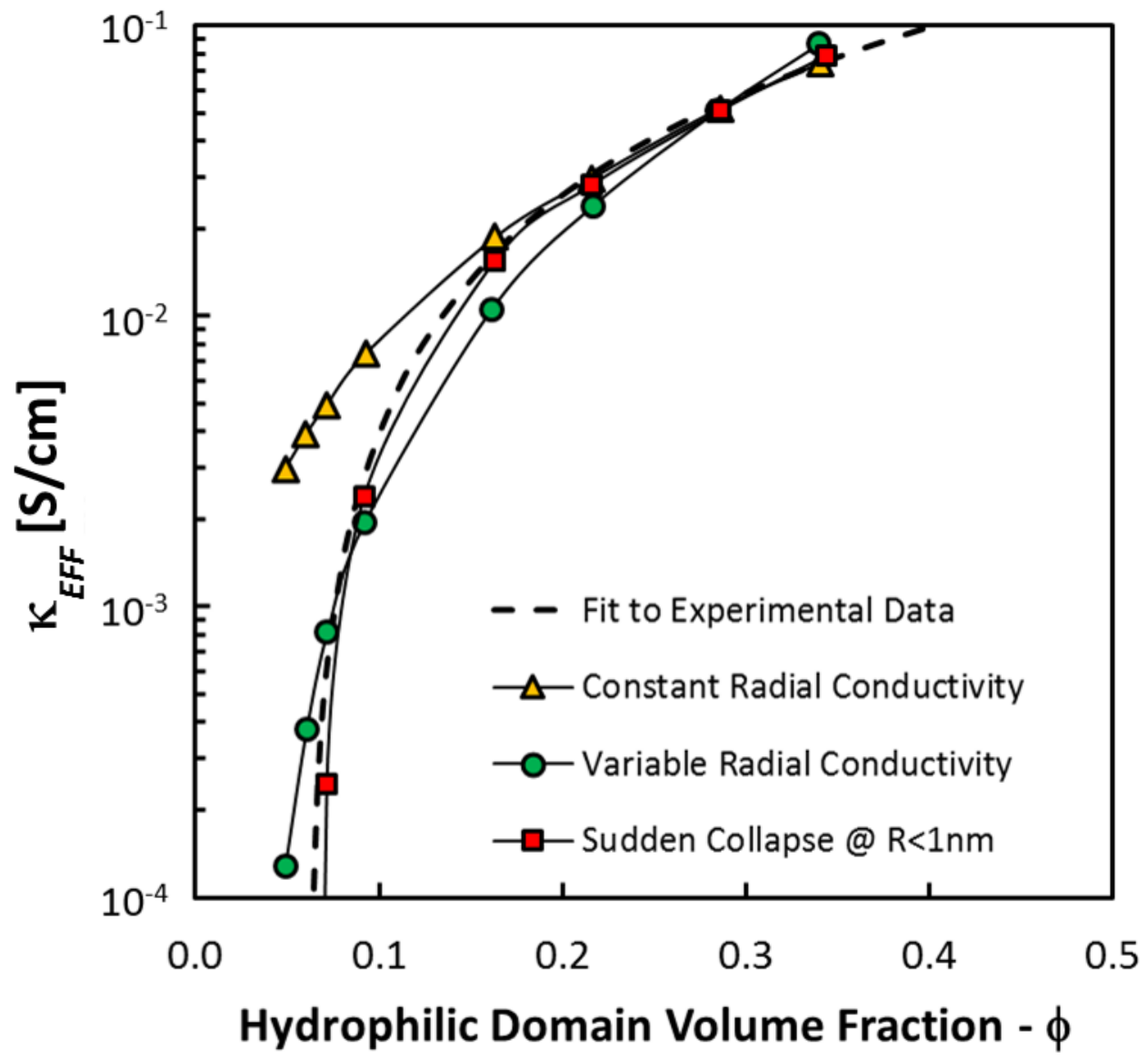

Figure 10: Nafion conductivity as a function of $\phi$ for various scenarios of throat conductivity. 


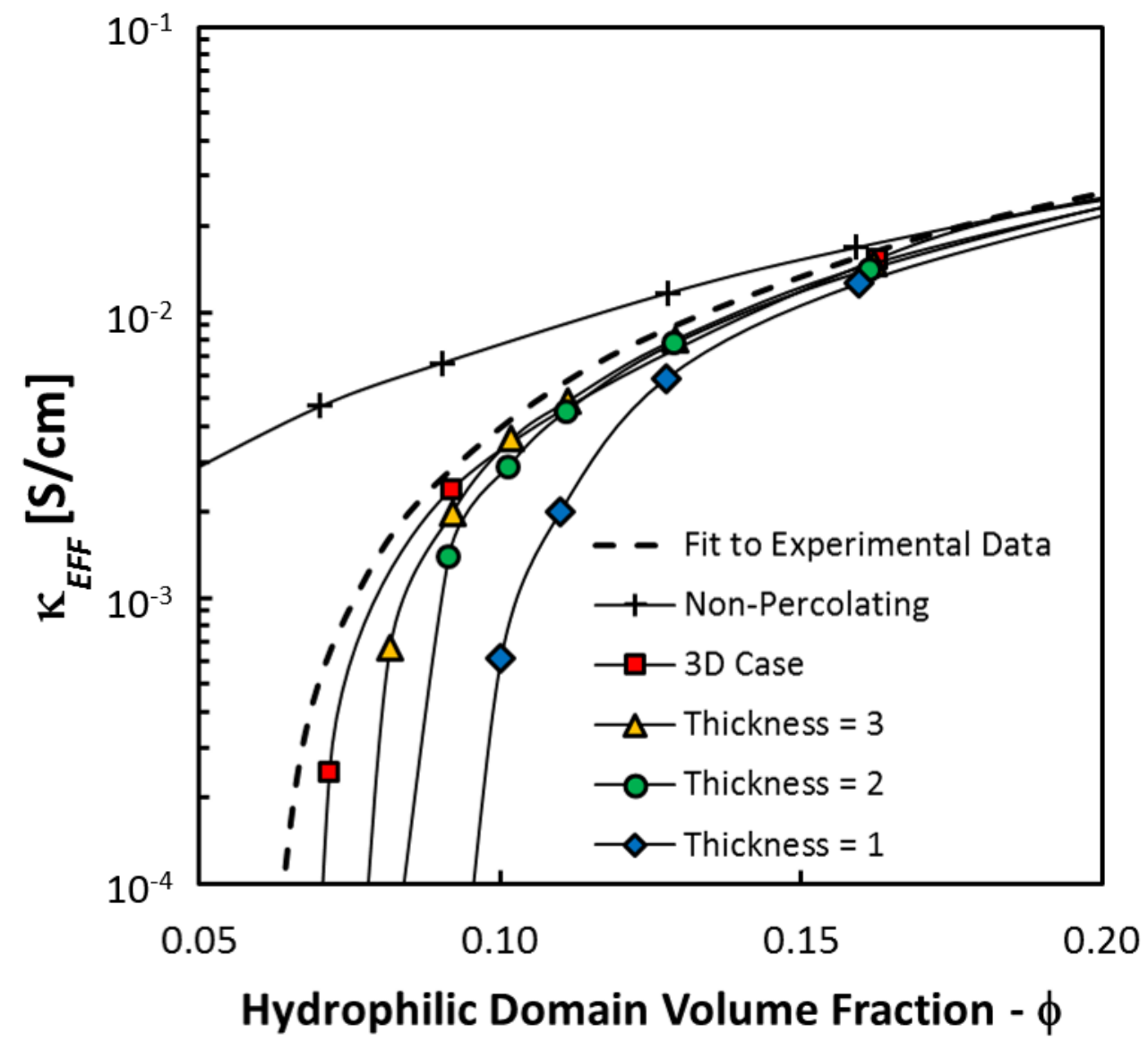

Figure 11: In-plane conductivity of Nafion thin films, with thickness indicated by the number of layers in the direction perpendicular to the current flow. Each layer of nodes represents about $16 \mathrm{~nm}$. 
Graphical Abstract:

Resistor-Network Modeling of Ionic Conduction in Polymer Electrolytes

Jeff T Gostick and Adam Z Weber

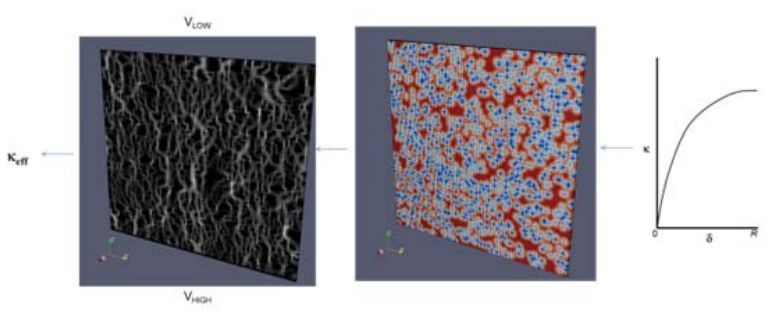

